\title{
Une diversité de configurations d'apprentissage en situation de travail pour réduire l'usage des engrais et pesticides agricoles
}

A diversity of learning configurations in work situations to reduce the use of agricultural fertilizers and pesticides

\section{Émilia Chantre, Marianne Le Bail et Marianne Cerf}

\section{OpenEdition}

Journals

Édition électronique

URL : http://journals.openedition.org/activites/1061

DOI : 10.4000/activites. 1061

ISSN : $1765-2723$

Éditeur

ARPACT - Association Recherches et Pratiques sur les ACTivités

\section{Référence électronique}

Émilia Chantre, Marianne Le Bail et Marianne Cerf, « Une diversité de configurations d'apprentissage en situation de travail pour réduire l'usage des engrais et pesticides agricoles », Activités [En ligne] 11-2 | Octobre 2014, mis en ligne le 15 octobre 2014, consulté le 19 avril 2019. URL : http:// journals.openedition.org/activites/1061; DOI : 10.4000/activites.1061

\section{(c) $\oplus \Theta \Theta$}

Activités est mis à disposition selon les termes de la licence Creative Commons Attribution - Pas d'Utilisation Commerciale - Pas de Modification 4.0 International. 


\title{
Une diversité de configurations d'apprentissage en situation de travail pour réduire l'usage des engrais et pesticides agricoles Émilia Chantre
}

UMR 1048 INRA-AgroParisTech SAD-APT, 16 rue Claude Bernard, 75231 Paris cedex 05, emilia.chantre@developpement-durable.gouv.fr

\section{Marianne Le Bail}

UMR 1048 INRA-AgroParisTech SAD-APT, 16 rue Claude Bernard, 75231 Paris cedex 05, lebail@agroparistech.fr

\section{Marianne Cerf}

UR 1326 INRA-SenS, 16 rue Claude Bernard, 75231 Paris cedex 05, cerf@agroparistech.fr

\begin{abstract}
A diversity of learning configurations in work situations to reduce the use of agricultural fertilizers and pesticides. The need to develop a more ecological agriculture is a strong injunction in current territorial policies, which raises questions about farmers' learning conditions in their work. Our methodology, at the interface between professional didactics and agronomy, is based on interviews with twenty French field crop farmers who have reduced their use of pesticides and fertilizers. To analyze the configuration of learning conditions under which each change in production methods was made, we have developed an analytical framework that distinguishes three stages (warning sign, experiencing, and evaluating), drawing inspiration from Dewey's theory of inquiry (1993). Each stage of the learning process has been characterized by a set of variables, themselves characterized by a set of modalities. Hierarchical clustering serves to identify ten types of configuration of learning conditions. Throughout their careers, farmers mobilize several types of configuration involving various forms of experience and resource persons. Even though farmers have tended to change their practices when in contact with others, some types of configuration have become completely autonomous (20\% of cases). This reveals both the isolation of some farmers and the ability of others to combine group learning and autonomous learning. Our identification of configurations of learning conditions is an original approach to addressing learning in working situations and provides tools to help farming consultants support shifts towards innovative cropping systems.
\end{abstract}

Keywords

learning conditions, farmers, changes in practices, inquiry

\section{Introduction}

L'actualité met sur le devant de la scène la nécessité, pour l'agriculture, de repenser ses modes de production pour réduire son impact sur l'environnement tout en assurant la sécurité alimentaire. En particulier, la transformation des modes de production utilisant de façon intensive des intrants (produits phytosanitaires, fertilisants azotés) est de plus en plus à l'ordre du jour avec le Grenelle de l'Environnement et son plan Ecophyto 2018 (injonction 
de réduction des produits phytosanitaires de $50 \%$ à l'horizon 2018) même si depuis 2013, les ambitions ont été revues à la baisse («Ecophyto, réduire et améliorer l'utilisation des phytos »).

Les enjeux sont à la fois environnementaux et sanitaires. Sur le plan de la santé, des travaux comme ceux de Garrigou, Baldi, \& Dubuc (2008) ont très tôt souligné les problèmes liés aux protections utilisées. Ils ont alerté sur ces questions, même s'il existe souvent un déni au sein même de la profession agricole et une mise en invisibilité des problèmes de santé liés à l'usage des pesticides (Jouzel \& Dedieu, 2013). Sur le plan environnemental, les agronomes ont montré que la réduction de l'usage des pesticides à hauteur de $50 \%$ suppose une reconception et la mise en place de pratiques permettant de valoriser les régulations propres à un agroécosystème (Jacquet, Butault, \& Guichard, 2011). En effet, si l'agriculture intensive en intrants a permis d'homogénéiser les situations agricoles et de standardiser les problèmes liés à la conduite des cultures (Chevassus-au-Louis \& Griffon, 2008), la réduction de leur usage implique une dépendance plus grande aux dynamiques agro-écologiques spécifiques à un contexte local dont il s'agit d'ailleurs de préciser les contours. Ces évolutions doivent se penser en lien avec l'activité et le travail agricole. Comme le notent Béguin, Dedieu et Sabouri (2011), « on peut [...] affirmer qu'il n'y a d'innovation dans le secteur qu'à la hauteur des transformations possibles ou impossibles du travail lui-même ». Néanmoins, à notre connaissance, les dynamiques entre innovation et travail dans le secteur agricole ont peu été abordées par des ergonomes jusqu'ici malgré quelques exceptions (Coquil, 2014 ; Jourdan, 1997 ; Nicourt \& Cabaret, 2011).

Dans cette perspective, nous souhaitons contribuer à étudier comment les agriculteurs s'engagent dans l'exploration et la mise en œuvre de solutions leur permettant de réduire l'usage des intrants (engrais et pesticides en particulier). Pour aborder ces processus de «problémation» ou de problématisation (Fabre \& Vellas, 2006) associés à la mise en place de pratiques réduisant l'usage des intrants, nous avons choisi, comme suggéré par (Cerf, Gibbon, Hubert, Ison, \& Jiggins (2000), de nous interroger sur la démarche d'enquête (au sens de Dewey (1993) que les agriculteurs réalisent à l'occasion des changements de pratique qu'ils opèrent. Il s'agit de comprendre comment les agriculteurs construisent leur expérience et l'énoncent, en partie en continuité, en partie en rupture, avec un mode de production basé sur l'utilisation intensive d'intrants. En procédant ainsi, l'idée est avant tout de pouvoir appréhender la façon dont un agriculteur, pour faire face à des mutations de son milieu professionnel et pour développer des pratiques dites écologisées, prend appui sur des ressources présentes ${ }^{1}$ dans son environnement social, technique, économique, législatif, mais aussi sur ses expériences passées. Il s'agit de reconnaître les situations ordinaires ${ }^{2}$ de travail comme des situations potentielles de développement ${ }^{3}$. En d'autres termes, il s'agit de participer à une organisation des situations de travail pour favoriser les processus d'enquête des agriculteurs pour qu'ils puissent agir dans l'incertain (Darnhofer, Bellon, Dedieu, \& Milestad, 2010) et s'adapter à l'évolution de leur environnement (évolution de l'environnement naturel et des conditions socio-économiques et réglementaires d'exercice de leur métier d'agriculteur). S'adapter ici ne doit pas se comprendre comme une simple réaction à des changements de l'environnement. Il s'agit également d'une anticipation de changements à venir. C'est bien là aussi que les apports du pragmatisme ${ }^{4}$ et plus

1 Notons que ces ressources peuvent évoluer au fil du temps en particulier celui de la carrière professionnelle d'un agriculteur, qui est la durée sur laquelle porte notre étude.

2 La notion de situation est à comprendre ici comme l'ensemble des circonstances dans lesquelles une personne se trouve, et des relations qui l'unissent à son milieu (Brousseau, 1998).

3 Mayen (1999) parle de «situation potentielle de développement » pour rendre compte « de l'ensemble des conditions qu'une situation doit remplir pour engager puis étayer le processus de développement des compétences d'un individu ou groupe d'individus ».

4 Deux numéros de la revue Activités ont particulièrement traité d'approches dites pragmatiques. Le volume 1, $\mathrm{n}^{\circ} 2$ sur l'action située, le volume $10, \mathrm{n}^{\circ} 2$ sur la pragmatique. Les travaux sur le cours d'action s'inscrivent aussi dans ces approches, en mobilisant un auteur comme Peirce plutôt que Dewey. 
particulièrement de la théorie de l'enquête de Dewey, que nous mobilisons dans ce travail, prennent tout leur sens selon nous.

Cet article ${ }^{5}$ propose de caractériser les configurations de conditions d'apprentissage que les agriculteurs évoquent pour expliciter la façon dont ils puisent dans les situations ordinaires de travail, les ressources qui leur permettent de mettre en place de nouveaux modes de conduite des cultures. Pour cela, nous proposons dans un premier temps un cadre d'analyse des processus d'enquête mis en œuvre par les agriculteurs pour s'engager et mettre en œuvre un changement de pratique. Pour nous, ce processus d'enquête est au fondement de l'apprentissage dans les situations ordinaires de travail sans néanmoins préjuger à ce stade qu'il engendre systématiquement des apprentissages que nous n'examinerons pas précisément dans cet article. Nous appliquons le cadre construit à un ensemble de récits faits par un échantillon d'agriculteurs d'une petite région à propos de leur trajectoire de changements de pratiques depuis le début de leur carrière professionnelle pour mettre en évidence une diversité de «configurations de conditions d'apprentissage» telles qu'elles émergent de leurs récits.

\section{1.- Analyser des configurations de conditions d'apprentissage des agriculteurs}

Face aux mutations que connaît un secteur d'activité, certains professionnels parviennent à réélaborer les compétences acquises, à les recombiner et à opérer des ruptures sur fond de continuité (Mayen, 2008). Qu'en est-il dans le secteur agricole et l'évolution des façons de conduire les cultures?

Dans le secteur agricole, depuis les années 1990, certains agriculteurs ont réussi à réduire l'usage des intrants (engrais, produits phytosanitaires en particulier) par rapport à ce qu'ils pratiquaient dans le cadre d'une agriculture qualifiée d' «intensive » ou de «conventionnelle» opposée à d'autres formes d'agricultures plus économes en intrants comme l'agriculture biologique, l'agriculture «intégrée», ou encore "autonome et économe » pour reprendre des termes circulant dans le monde agricole. Cette construction s'est opérée sur le temps long et le résultat observé aujourd'hui est le fruit de nombreux petits changements apportés dans la façon de produire des cultures (Coquil, 2014 ; Lamine, 2011). Ainsi, ces agriculteurs ont élaboré, au fil de leur carrière professionnelle, des modes de conduite des cultures qui contrastent, en termes de recours aux intrants azotés et phytosanitaires, avec ce que font aujourd'hui la majorité des agriculteurs.

\section{1.- L'enquête au cœur du développement en situation ordinaire de travail ?}

Dans un contexte où peu de travaux de recherche et peu de structures de conseil s'intéressent concrètement à la façon de réduire l'usage des intrants, les agriculteurs n'ont pas appliqué des modes de conduite des cultures préconstruits par la $R \& D$ ou le conseil agricole. Il s'agit bien d'un processus de changement qui s'opère au cœur de la dynamique et de l'indétermination des situations auxquelles ces professionnels ont à faire face et du couplage qu'ils opèrent avec leur environnement pour agir dans et sur celui-ci.

Le couplage entre un sujet, ses connaissances, son activité et son environnement fait l'objet de différents cadres théoriques et de débats entre les tenants d'une approche cognitiviste, ceux d'une cognition située et distribuée, ou encore ceux d'une approche pragmatique. De la même façon, une diversité d'approches traite des conditions dans lesquelles des individus ou des collectifs développent des compétences ou des capacités pour opérer ce couplage et agir efficacement en situation. Rogalski (2004) donne un aperçu de ces débats entre des approches cognitivistes et des approches situées, et met en avant la façon dont, à ses yeux, la didactique professionnelle constitue «une théorie de la formation et du développement des

Ce travail a bénéficié du soutien financier de l'ANR (Projet Popsy ANR 08-STRA 12). 
compétences professionnelles - théorie liée à une théorie de l'activité dans le champ de la psychologie ergonomique - qui ne cherche pas à faire du passé table rase » (p.117). Cependant, la didactique professionnelle a été construite dans un rapport de continuité avec les approches de l'activité qui s'appuient sur la distinction entre tâche et activité. De ce fait, elle n'est pas nécessairement en mesure de rendre compte de la façon dont se développe l'activité de professionnels pour qui les prescriptions sont multiples et pour lesquels le poids des normes professionnelles élaborées dans des collectifs de pairs (Darré, 1985) est sans doute clé, comme c'est le cas pour l'agriculture.

Comme le suggère Mayen (2008), «il est possible d'identifier les conditions du développement fournies par les situations de travail avec l'idée que la confrontation aux problèmes n'est pas la seule source du développement, que des configurations faisant converger trajectoires individuelles et trajectoires des situations, problèmes à résoudre et ressources sociales, en constituent d'autres sources». Ainsi, pour saisir comment un agriculteur explore de nouvelles pratiques, et développe son activité, ne faut-il pas s'interroger sur le potentiel de développement des situations et plus précisément aux «modalités organisées pour apprendre, trouver des ressources pour agir et pour résoudre des problèmes inédits, autrement dit, [aux moyens dont le professionnel compétent] dispose pour apprendre de son expérience et apprendre de son environnement»? Pour évoquer ces modalités organisées, Mayen (ibid.) parle de «stratégie». En retenant la notion d'enquête proposée par Dewey (1993), nous souhaitons remettre ces stratégies en lien avec l'expérience que les agriculteurs font de leur environnement, la situation.

De fait, selon Dewey (2004), la situation est l'environnement dont le sujet fait l'expérience. Elle n'est pas un «donné », mais un « construit » lié à un projet d'adaptation du sujet à son environnement, projet plus ou moins distancié d'une adaptation strictement fonctionnelle et produit d'expériences antérieures réussies. Le processus qui permet de réduire l'indétermination d'une situation pour permettre de définir une conduite ajustée et de résoudre le ou les problèmes rencontrés dans la situation, de faire face à une situation problématique, est l'enquête. Dewey (1993) la définit comme «la transformation contrôlée et dirigée d'une situation indéterminée en une situation qui est si déterminée en ses distinctions et relations constitutives qu'elle convertit les éléments de la situation originelle en un tout unifié ». L'enquête est ce qui permet non seulement de résoudre les problèmes, mais aussi de les constituer comme tels par le sujet. Ainsi, Dewey (2004) distingue plusieurs phases: la perception du problème, sa détermination ou construction, la suggestion de solutions possibles, l'examen raisonné des suggestions et de leurs conséquences, le test des hypothèses. Mais l'enquête s'inscrit plus largement dans une «théorie du jugement » qui permet à Dewey de revisiter la logique propositionnelle comme le soulignent Fabre et Vellas (2006).

Le processus d'enquête repose sur l'élaboration d'assertions dont la véracité et la pertinence sont «garanties » par les règles du processus d'enquête. Pour arriver à quelque chose de suffisamment assuré pour supporter l'action, l'enquête passe par toute une série de phases, des premières propositions, aux affirmations intermédiaires plus crédibles, plus robustes, jusqu'aux « jugements » finaux qui posent la détermination de la situation. Comment s'opère cette construction progressive ? Il s'agit de comprendre comment sont mis à l'épreuve des faits et les modes de qualification des objets d'un côté, et comment interviennent des systèmes de concepts et d'instruments dans cette qualification de l'autre. L'enquête existe et vit par les règles qui la gouvernent, incluant l'évolution éventuelle de ces règles sous l'effet de l'enquête elle-même.

Nous présenterons ainsi la façon dont les agriculteurs arrivent à juger que tel ou tel changement technique est acceptable et que «ça marche », et la façon dont éventuellement évoluent leurs façons d'en juger au cours de leur carrière professionnelle. Nous ne faisons pas état ici des changements qui s'opèrent au niveau cognitif ou normatif, résultats présentés par ailleurs (Chantre, Le Bail, \& Cerf, 2013). Ainsi nous examinons ici, en nous inspirant de l'analyse faite par Fabre \& Vellas (2006) :

— la convenance fonctionnelle ou opérationnelle d'une solution, 
— l'intégration dans un système de significations «déjà là » comme le propose Dewey (ibid.),

— la révision des conditions de validité des inférences faites,

— le travail nécessaire pour éventuellement hiérarchiser ou trancher entre des conditions qui peuvent s'avérer contradictoires dans leurs conséquences et qui peuvent s'avérer clés dans des processus de problématisation.

Nous proposons un cadre pour identifier, selon les phases de l'enquête, différents attributs des situations qui contribuent à dessiner des « configurations de conditions d'apprentissage, et des situations potentielles de développement» (Mayen, 2008). S'intéresser à ces configurations revient pour nous à considérer comment, dans les phases de l'enquête, s'articulent, pour l'agriculteur, d'une part la confrontation aux situations problématiques qu'il rencontre dans l'exercice de son activité au sein de son exploitation et, d'autre part, « la confrontation à l'usage des produits de la culture, à son activité inscrite dans un univers social constitué d'artefacts, fournissant des modalités pour construire et faire évoluer l'organisation de l'activité elle-même » (Mayen, ibid.).

\section{1.-2.- De l'enquête aux configurations de conditions d'apprentissage}

Comme Mayen (ibid.) «nous soutenons l'hypothèse selon laquelle un grand nombre de formes sociales et plus précisément d'interactions sociales jouent, dans la vie professionnelle, un rôle d'étayage, au sens que donne Bruner à ce terme (Bruner, 1983, 2000). Rôle d'étayage pour l'apprentissage avant, $[\ldots]$ pendant et après, parce que certaines interactions contiennent un grand nombre d'ingrédients propres à constituer des situations dans lesquelles tout individu peut apprendre des situations. Celles-ci étant toujours des situations tissées de rencontres avec d'autres, apprendre des situations, c'est, en grande partie, apprendre des autres ».

En phase avec cette position, les travaux dans le monde agricole ont depuis longtemps mis en avant l'importance de ces interactions sociales dans la construction de normes professionnelles dans des collectifs aux configurations variées (Darré, 1985 ; Goulet, 2008 ; Lamine, Meynard, Perrot, \& Bellon, 2009). Le réseau, la communauté de pratiques, le groupe professionnel local, tels que les développent ces auteurs, sont des lieux où s'articulent des connaissances issues de «mondes professionnels » différents (au sens de Béguin (2004)) où s'élaborent des significations pour appréhender les objets et les phénomènes sur lesquels agir, et où se construit parfois une identité professionnelle.

Néanmoins, nous faisons l'hypothèse que la construction de la signification de nouvelles pratiques qui s'opère au sein de ces groupes, réseaux ou communautés n'est pas nécessairement suffisante pour permettre à l'agriculteur de construire des significations renouvelées pour agir face aux situations problématiques et singulières qu'il rencontre, et pour inscrire les transformations de pratiques qu'il opère dans son histoire singulière et son expérience. Ces genèses opératives et identitaires, pour reprendre les termes de Pastré (2005), si elles s'opèrent dans des interactions sociales variées, ne sont-elles pas soutenues par ce que Jourdan (1997) décrit comme des procédures de recherche, d'expérimentation, de validation qui s'avèrent elles-mêmes appuyées par des observations ou des échanges avec des pairs ou des conseillers agricoles?

Lamine et al. (2009) évoquent également l'existence de ces essais dans des trajectoires de conversion vers l'Agriculture Biologique dites «préparées» et de nombreux travaux soulignent que «l'expérimentation à la ferme » est inhérente au métier d'agriculteur luimême (Bentley, 2006 ; Hocdé \& Triomphe, 2006 ; Rhoades \& Bebbington, 1991). Pour ces auteurs, elle constitue l' " une des stratégies fondamentales dans la tentative des agriculteurs pour connaître et contrôler leur environnement » (Berkes \& Turner, 2006). Il ne faut pas entendre ici «expérimentation » comme une modalité de construction d'un fait scientifique. Il faut la comprendre comme une opération contrôlée par des règles plus ou moins explicites permettant de faire l'expérience d'un couplage réussi entre une conduite adoptée et un environnement, et de conclure sur la possibilité de la réussite renouvelée de ce couplage. 
C'est pourquoi nous parlerons plutôt par la suite de « formes d'expérience ».

En suivant Jourdan (ibid.) et Kolb (1984), une forme d'expérience s'opère suite à l'identification d'un problème. Mais qu'est-ce qui constitue un problème, ou plus exactement une situation problématique, pour l'agriculteur? Est-ce uniquement une confrontation à une situation de production dans laquelle il n'arrive pas à obtenir les résultats qu'il souhaite, ou bien est-ce aussi l'anticipation des évolutions d'un contexte et les risques qu'il perçoit à ne pas faire évoluer ses pratiques pour faire face à des mutations en cours ? Ainsi, le champ de ce qui constitue une situation problématique pour les agriculteurs s'étend au-delà des difficultés rencontrées dans la conduite sensu stricto des cultures, et concerne plus largement sa façon d'intégrer, dans ses actes productifs, l'ensemble des évolutions socio-économiques et techniques.

Dans ce qui suit, nous proposons dès lors d'appréhender les configurations de conditions d'apprentissage comme des combinaisons d'interactions sociales et de formes d'expériences, organisées en un processus composé de phases successives. Les agriculteurs puisent des ressources, que ce soit pour identifier ou construire des problèmes, des solutions, les évaluer et construisent des formes d'expériences, qui sont l'occasion de tester et d'évaluer ces solutions dans le contexte singulier de leur propre activité ou dans un contexte proche dans le cas de l'observation chez un pair. Nous proposons donc d'analyser ces combinaisons en distinguant trois phases successives qui renvoient aux processus d'enquête et de construction du jugement proposés par Dewey (1993) et qui constituent pour nous une façon d'approcher le processus d'apprentissage d'une nouvelle pratique (voir Tableau 1) :

1. la première phase dite de «mise en alerte » correspond à l'émergence d'une idée, soit en réaction à un événement lié à des dynamiques internes (en particulier au niveau de l'agroécosystème, mais aussi lors d'une évolution de la main-d'œuvre disponible par exemple) ou externes (en particulier liées à l'évolution du contexte économique, mais aussi aux débats sur l'agriculture dans la société par exemple), soit en lien avec une veille économique, réglementaire ou technique que l'agriculteur réalise. Cette phase correspond aux trois étapes de perception du problème, de sa détermination ou construction, et de la suggestion de solutions possibles telles que Dewey (1993) les a précisées ;

2. la deuxième, ou «phase d'expérience», permet d'expérimenter l'idée retenue et correspond à l'examen raisonné des suggestions et de leurs conséquences ;

3. la troisième ou «phase d'évaluation », vise à confronter les résultats obtenus grâce à la pratique expérimentée à un référentiel (interne issu de sa propre expérience ou/et externe) qui se décline selon des critères d'évaluation variables, et correspond à la phase de test des hypothèses qui permet la construction du jugement puisqu'à l'issue de cette phase, les agriculteurs peuvent ou non adopter cette pratique.

\begin{tabular}{|l|l|}
\hline \multirow{4}{*}{ 1. Phase de mise en alerte } & $\begin{array}{l}\text { Contextes : contexte politico-économique macro, dispositif politico- } \\
\text { économique territorialisé }\end{array}$ \\
\cline { 2 - 2 } & Préoccupation interne \\
\cline { 2 - 2 } & Anticipation d'un problème ou réponse à un problème observé \\
\cline { 2 - 2 } & Ressource intervenant : autrui, autre (presse par exemple) \\
\cline { 2 - 2 } & Evènement déclencheur de l'expérience \\
\hline \multirow{2}{*}{$\begin{array}{l}\text { «. Phase d'expérimentation » } \\
\text { 3. Phase d'évaluation }\end{array}$} & Déroulement opérationnel \\
\cline { 2 - 2 } & Réalisation de l'essai avec autrui \\
\cline { 2 - 2 } & Critères \\
\cline { 2 - 2 } & Référentiel interne \\
\cline { 2 - 2 } & Référentiel externe \\
\cline { 2 - 2 } & Changement adopté/non \\
\hline
\end{tabular}

Tableau 1 : Les phases successives d'un processus d'apprentissage d'une nouvelle pratique, et les variables retenues pour les caractériser à partir des récits des agriculteurs de notre échantillon

Table 1: Successive phases of the process of learning a new practice, and variables retained to characterize them, based on our interviews with farmers 


\section{2.- Construire des configurations de conditions d'apprentissage pour la réduction de l'usage des intrants}

La réduction de l'usage des intrants a rarement lieu de façon radicale chez les agriculteurs. Lamine et al. (2009), qui ont étudié les trajectoires de changement des agriculteurs ayant choisi de se convertir à l'agriculture biologique, mettent en avant que les trajectoires les plus robustes sont celles qui s'opèrent dans la durée et au sein desquelles la conversion vers l'agriculture biologique s'inscrit dans un processus d'évolution antérieure des pratiques. Le même constat est fait pour des agriculteurs qui se tournent vers la production intégrée telle qu'elle s'est développée à partir des travaux sur les blés à bas niveau d'intrants. Dès lors, le recueil de données ne peut pas s'opérer uniquement à partir d'une observation en situation de travail.

\section{1.- Réduire les intrants : un changement qui s'inscrit dans la durée d'une carrière professionnelle}

Nous avons ainsi fait le pari de retracer la succession de changements que les agriculteurs ont réalisée depuis le début de leur carrière professionnelle et de reconstituer la trajectoire de changement de pratiques suivie par chacun de ces agriculteurs (Chantre, 2011). La reconstruction des trajectoires de changement s'appuie sur l'identification de ce que nous avons appelé des «pratiques de transition» (Chantre, Cerf, \& Le Bail, 2014), pratiques qui, pour les agronomes, marquent effectivement une modification des objectifs visés et/ou des modalités de mise en œuvre de techniques de cultures. Ces pratiques reflètent donc, pour l'agronome, une évolution dans la façon de construire le rapport entre l'action sur l'agroécosystème et la dynamique propre de ce dernier. Sur cette base, nous nous sommes alors intéressées aux ressources que les agriculteurs ont mobilisées pour acquérir la connaissance et/ou la maîtrise d'une pratique de transition donnée, ou pour tester une pratique qu'ils ont ensuite abandonnée.

Concrètement, nous avons réalisé des entretiens semi-directifs en 2009-2010 auprès de vingt céréaliers français en Champagne Berrichonne de l'Indre, sélectionnés parce qu'ils sont identifiés par leurs pairs ou par des conseillers ${ }^{6}$ de leur région comme ayant des niveaux d'utilisation des pesticides et de l'azote plus faible que ceux constatés en général dans la région, ce que les enregistrements de pratiques collectés chez ces agriculteurs confirment (Chantre et al., 2014). Lors d'un premier entretien, nous avons invité l'agriculteur à retracer l'ensemble des changements survenus dans son exploitation depuis le début de sa carrière professionnelle (dans l'échantillon, cela représente une période allant de 1975 pour l'installation la plus ancienne à 1998 pour la plus récente), que ces changements concernent ou non les pratiques de transition que nous avons retenues par la suite et plus explicitement reliées à un changement de niveau d'intensification de l'usage des intrants. En effet, le champ cultivé et l'exploitation sont appréhendés ici comme des systèmes emboîtés et ouverts (systèmes de culture et système d'exploitation, Sebillotte (1974)) et les interactions au sein de ces systèmes, comme avec leur environnement, obligent à enquêter sur les changements qui influent, pour l'agriculteur, sur le fonctionnement et l'emboîtement de ces systèmes.

Nous avons alors construit une frise chronologique de ces changements ou trajectoire de changements de pratiques (un exemple est montré en Figure 1) qui a servi de base à un second entretien. Celui-ci nous a permis de valider avec l'agriculteur la succession ou concomitance des changements, mais aussi de lui faire expliciter les conditions dans lesquelles s'étaient opérés ces changements. Dans ce sens, notre démarche est proche de l'entretien d'explicitation (Vermersch, 2007) car elle vise à créer les conditions nécessaires pour que l'interviewé puisse rendre compte le plus finement possible de ce qu'il a fait

6 Dans la suite du texte, si la structure d'appartenance n'est pas précisée, nous désignons par conseiller agricole toute personne intervenant auprès des agriculteurs pour délivrer un conseil et dépendant soit de la Chambre d'Agriculture, soit d'une coopérative, d'un négoce, d'une association (CIVAM par exemple) ou encore travaillant à son propre compte (conseiller privé). 
réellement, de comment il s'y est pris pour faire ce qu'il avait à faire tout en ne cherchant pas à identifier précisément les prises d'information dans les situations évoquées mais plutôt la façon dont il s'y est pris pour s'impliquer dans un processus de changement.

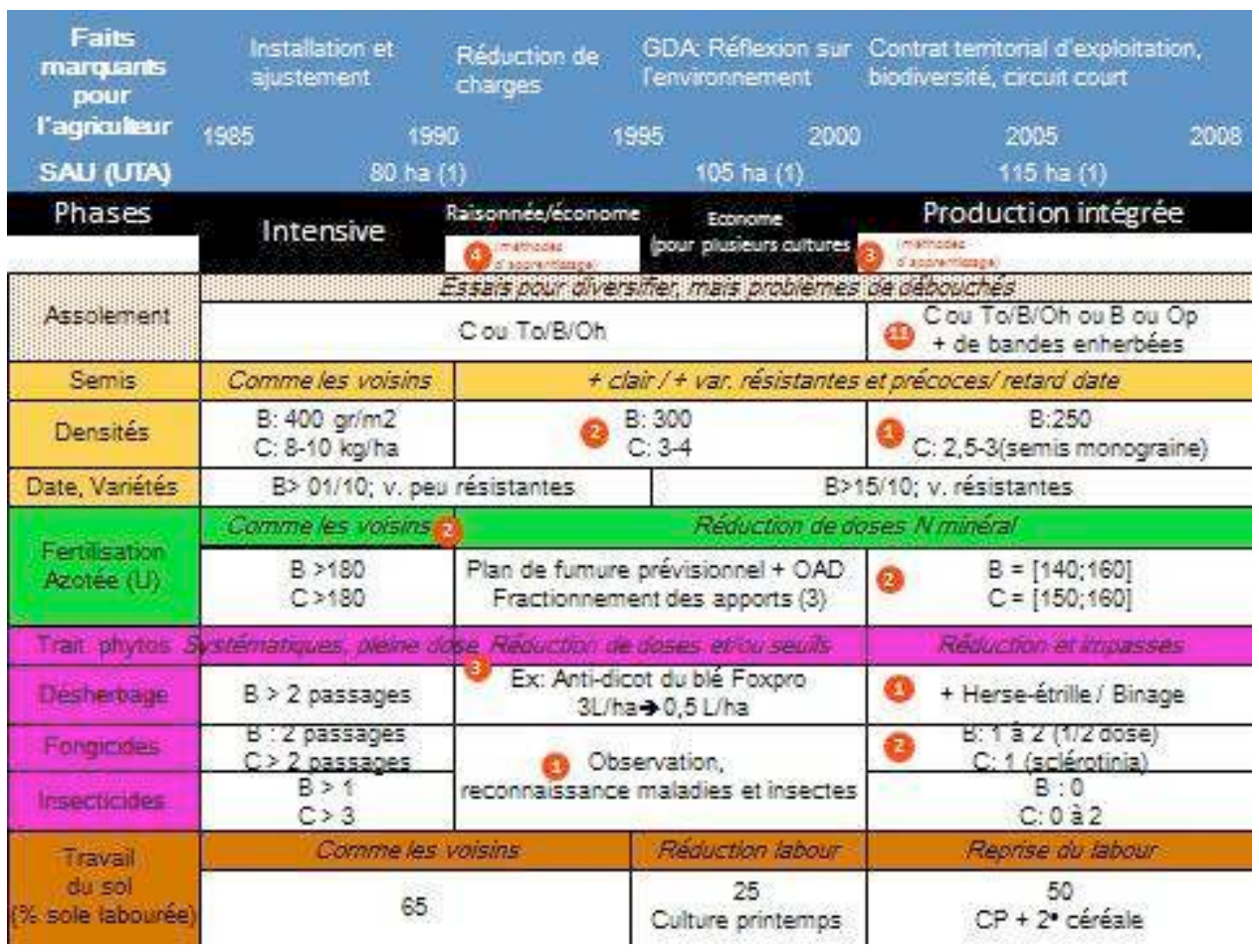

Figure 1 : Trajectoire de changements de pratiques et localisation des processus d'apprentissage dans le cas de l'agriculteur Thomas.

La présentation des trajectoires de changement que nous avons adoptée consiste à identifier des phases au cours desquelles une certaine cohérence agronomique entre les pratiques se dégage (les colonnes). Les types d'opérations techniques classiquement identifiées par les agronomes pour la conduite des cultures sont en ligne, et l'ensemble de ce qui spécifie une opération figure dans une même couleur. Pour chaque opération technique, nous nommons ce qui a changé au cours de la phase considérée, et nous détaillons les modalités de ce changement. Dans chaque rond rouge est précisé le nombre de récits que nous avons codés en processus d'apprentissage relatifs à des changements de pratiques. Certains processus d'apprentissage renvoient à la façon même d'apprendre à expérimenter ou évaluer des changements et sont notées « méthodes d'apprentissage » dans le schéma.

Abréviations : GDA : groupe de développement agricole ; ha : hectares ; SAU : surface agricole utile ; UTA : unité de travail annuel ; $B$ : blé ; $C$ : colza ; To : Tournesol ; Oh : Orge d'hiver ; Op : Orge de printemps.

Figure 1: Trajectory of changes in practices and localization of learning processes in the case of the farmer Thomas.

Our presentation of trajectories of changes in practices consists in identifying phases during which a certain agronomic coherence emerges within the practices (columns). The types of technical operations typically identified by agronomists for crop management are linear, and everything relating to a particular operation is in the same color. For each technical operation, we name what has changed during the phase considered, and we detail the modalities of this change. In the red circles we have specified the number of narratives we have coded as learning processes related to changes in practice. Some learning processes refer to the way of learning to experiment or to evaluate changes and are called "méthodes d'apprentissage" (learning methods) in the diagram.

Abbreviations: GDA: Farmers agricultural development group; ha: hectares; SAU: Utilized Agricultural Area; UTA: annual work unit; B: Wheat; C: rapeseed; To: Sunflower; Oh: Winter Barley; Op: Spring Barley.

L'encadré 1 donne un exemple de récit mis en forme avec la grille d'analyse présentée 
auparavant. L'exemple choisi a trait à la réduction de la fertilisation : il aurait pu tout à fait traiter de la réduction d'utilisation de pesticides.

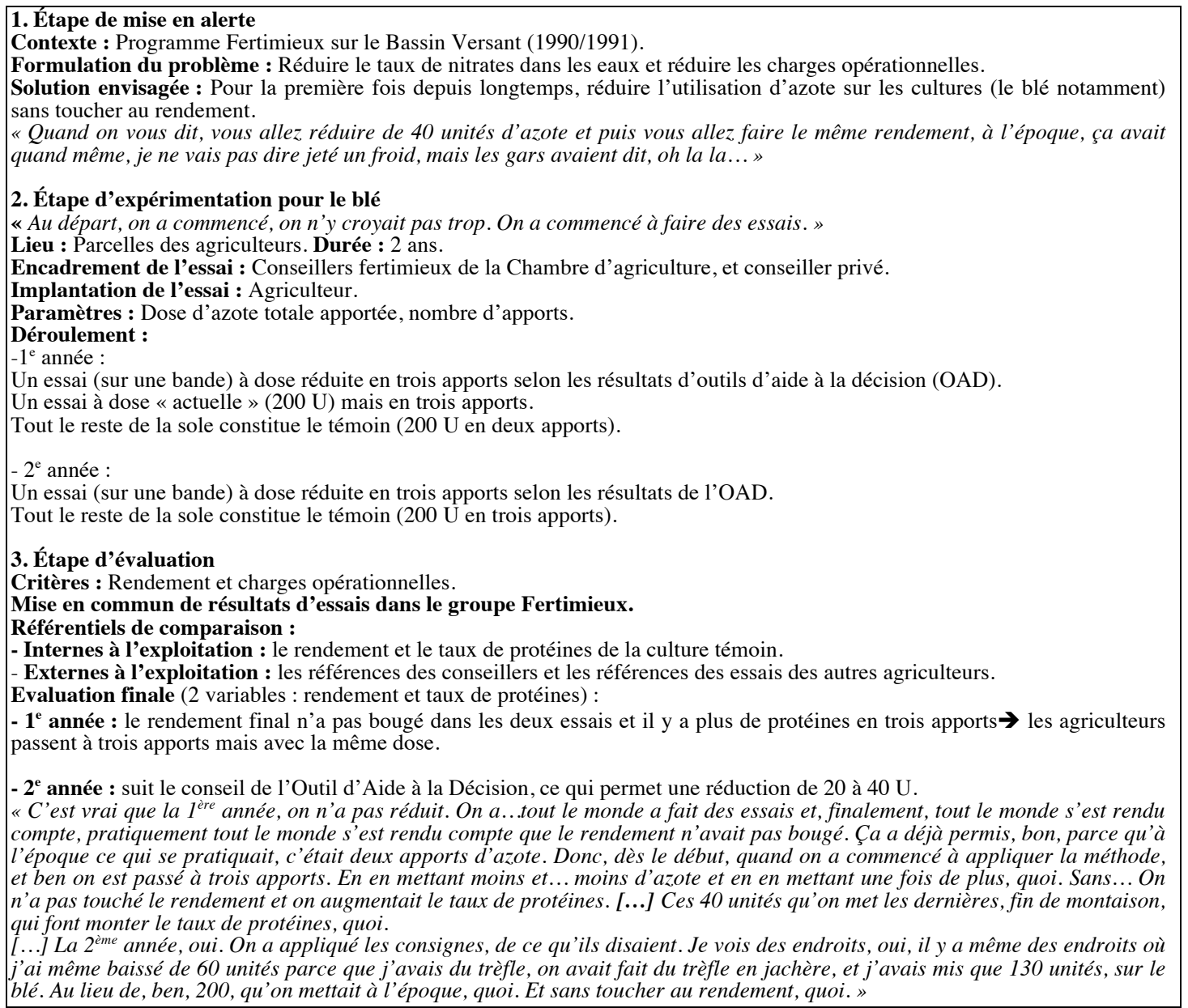

Encadré 1 : Récit de l'agriculteur Pierre au sujet du raisonnement de l'azote mis en forme selon la grille d'analyse

\section{Box 1: Farmer Pierre's narrative on nitrogen rationalization, formatted according to our analysis grid}

Nous avons procédé ainsi pour chaque changement réalisé au cours de la carrière professionnelle d'un agriculteur, et ce pour huit agriculteurs. Selon les agriculteurs, le nombre de récits produits pour décrire ce qu'ils ont mobilisé pour opérer des changements dans leur carrière professionnelle est variable (de 6 à 32 récits), ce qui correspond à une moyenne de 20 récits par agriculteur.

\section{2.- Construire des configurations de conditions d'apprentissage}

Pour huit agriculteurs, nous disposons ainsi d'une base de données de 156 récits qui ont donné lieu à un codage en double aveugle visant à appliquer le cadre d'analyse des processus d'enquête (Tableau 1). Ainsi, nous avons codé les phases qui le constituent (mise en alerte, «expérimentation », évaluation), les ressources mobilisées par les agriculteurs pour chacune de ces phases et d'autres variables que nous avons définies à la lecture de la base de données, pour appréhender la façon dont ces ressources sont mobilisées au cours de chaque phase. L'analyse de la base ainsi constituée nous conduit à proposer un nombre fini de variables et de modalités permettant de monter en généricité par rapport à la façon dont les agriculteurs parlent des ressources qu'ils mobilisent. Le tableau 2 présente les modalités que nous avons retenues pour chaque variable à partir de notre échantillon, et qui permettent ainsi de décrire de façon calibrée chaque récit. Ces modalités qualitatives sont soit directement identifiables à partir du discours de l'agriculteur, soit inférées à partir de ce qui est exprimé. Lorsque 
l'inférence n'était pas possible, la variable est considérée comme «non renseignée ».

Parmi les 156 récits ainsi formalisés, 36 présentaient au moins une des huit variables «non renseignée »: nous avons choisi de les éliminer pour éviter de voir une modalité «non renseignée " apparaître dans les résultats de l'analyse. La base de données est donc constituée d'une série de 120 processus d'apprentissage rapportés chacun à une tentative de changement de pratique d'un agriculteur, décrits par un jeu de huit variables descriptives (Tableau 2, en annexe) qui sont toutes qualitatives (hormis la variable durée du processus d'apprentissage).

Nous avons alors procédé à un traitement statistique, la classification ascendante hiérarchique $(\mathrm{CAH})^{7}$ pour identifier des «configurations types de conditions d'apprentissage », c'est-à-dire des configurations caractérisées par des modalités particulières à chaque phase (mise en alerte, expérience, évaluation) et qui peuvent donc être communes à plusieurs agriculteurs. Nous avons choisi de retenir un niveau d'agrégation de 10 classes qui apparaît suffisant pour décrire la diversité de notre base de données sans en perdre la richesse ${ }^{8}$. À partir des variables qui décrivent le mieux les classes, nous caractérisons ainsi dix configurations types de conditions d'apprentissage.

\section{3.- Résultats}

\section{1.- Interactions sociales et formes d'expérience : quelques constats au sein de l'ensemble des configurations de conditions d'apprentissage étudiées}

Nous proposons tout d'abord de regarder l'ensemble des récits mis en forme selon notre grille en nous focalisant sur les variables «interaction avec autrui » et «déroulement de l'expérience » puisque nous appréhendons les configurations de conditions d'apprentissage comme des combinaisons d'interactions sociales et de formes d'expériences.

L'analyse de la répartition des modalités des variables «interaction avec autrui » met en évidence tout d'abord que l'interaction sociale peut être différente dans la phase de mise en alerte et la phase d'expérimentation et cela que ce soit au sein de l'ensemble de la base (Figure 2) ou pour une même configuration de conditions d'apprentissage en particulier pour les configurations 4, 8 et 9 (voir Tableau 3 de la partie suivante). Ensuite, elle montre qu'une diversité d'interactions sociales intervient dans les configurations de conditions d'apprentissage : l'intervention individuelle d'un conseiller ou d'un pair lors de la phase de mise en alerte est plus fréquente que celle du groupe de développement ${ }^{9}$. De plus, une part non-négligeable des processus d'enquête s'effectue de façon isolée (modalité «non »), en particulier dans la phase d'expérience, qui dans plus de $50 \%$ des cas constitue une expérience uniquement personnelle. Ce dernier point nous conforte ainsi dans l'idée que la construction de la signification de nouvelles pratiques qui s'opère au sein de groupes, réseaux ou communautés n'est pas suffisante pour permettre la reconstruction de l'expérience.

7 La CAH a été réalisée à partir de la méthode de Ward en utilisant le logiciel R accompagné de FactoMineR (Husson, Lê, \& Pagès, 2009).

8 Le critère dominant de ce choix est l'interprétabilité des classes (Husson et al., 2009). Pour plus de détails sur la classification ascendante hiérarchique telle que nous l'avons utilisée, voir Chantre (2011).

9 Nous appelons groupe de développement agricole (GDA) dans le texte tout groupe d'agriculteurs réuni autour de questions agronomiques et animé par un conseiller ou animateur issu soit d'une Chambre d'agriculture, d'un CIVAM (Centres d'Initiatives pour Valoriser l'Agriculture et le Milieu rural) ou d'un CETA (Centre technique agricole). 


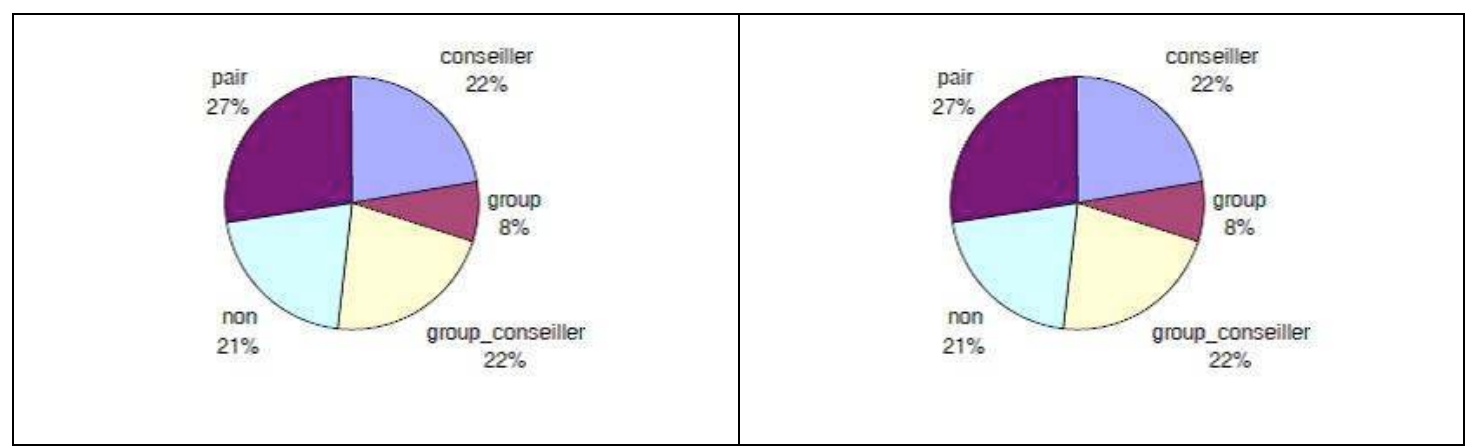

Figure 2: Répartition dans notre échantillon des modalités des variables « interactions avec autrui » dans la phase de mise en alerte (à gauche) et dans la phase d'expérience (à droite)

Légende : group : groupe de développement agricole; groupe_conseiller : conseiller intervenant dans un groupe de développement ; non : pas d'interaction avec autrui. Pour plus de détails, voir la description des modalités dans le tableau 2.

Figure 2: Distribution in our sample of the modalities of the "interaction with others" variable during the warning sign stage (left) and the experimenting stage (right)

Key: group: agricultural development group; groupe_conseiller: advisor involved in a development group; non: no interaction with others. For more details, see description of the modalities in Table 2

Les formes d'expérience (Figure 3) se déroulent à la fois d'une façon proche de l'expérimentation scientifique, appelée ici « comparaison synchronisée » $(39 \%)$, mais aussi de façon directe $(35 \%)$ : dans ce cas l'agriculteur met en place le changement directement sur toute la culture ou surface concernée et analyse les impacts après. L'expérience par analogie à ce qui se fait en dehors de chez soi (chez un pair, dans une station expérimentale), appelée «observation extérieure » est pratiquée dans moins de $10 \%$ des cas, comme s'il était plus convaincant de réaliser un test soi-même, chez soi, avant d'adopter une nouvelle pratique. Il ne s'agit pas ici de nier l'intérêt que portent les agriculteurs à des expérimentations et expériences faites ailleurs que dans leur exploitation, mais de mieux mettre en avant l'importance d'un processus qui s'apparente à la construction progressive d'une solution dans le cadre de l'enquête conduite par l'agriculteur.

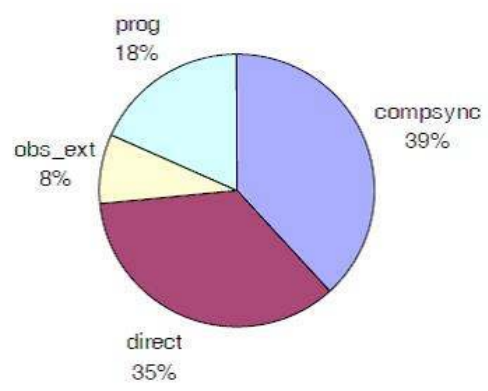

Figure 3 : Répartition dans notre échantillon des modalités de la variable « déroulement de l'expérience »

Légende: Expérimentation Prog: progressive; direct: directe; compsync: comparaison synchronisée. Obs_ext : observation extérieure.

Figure 3: Distribution in our sample of the modalities of the "course of the experiment" variable

Key: Expérimentation Prog: progressive; direct: direct; compsync: synchronized comparison; Obs_ext: external observation

À ce stade, il nous apparaît alors nécessaire de mieux identifier comment l'interaction avec autrui dans la phase de mise en alerte s'articule ou non avec l'interaction avec autrui dans la phase d'expérience, et pour finir avec la phase d'évaluation et la place d'un référentiel externe dans cette évaluation. 


\section{2.- Dix configurations-types de conditions d'apprentissage mobilisant différemment interactions sociales et formes d'expérience}

Les modalités qui distinguent le mieux les classes de configuration de conditions d'apprentissage, ou «configurations types » sont présentées dans le tableau 3 (en annexe). Les deux variables les plus discriminantes de la classification sont l'interaction avec autrui dans la phase d'expérience, et l'ensemble de critères d'évaluation de la phase d'évaluation.

Dans notre échantillon, il ne semble pas y avoir de configuration-type dominante. Les diverses formes d'interactions sociales sont relativement équilibrées entre les différentes configurations-types et elles se font avec :

- le groupe de développement agricole (GDA), qui comporte ou non un expert extérieur au groupe (configurations $n^{\circ} 1 \& 2$ ), quelle que soit la structure d'appartenance du conseiller ou du groupe,

- des pairs en dehors d'un groupe, voisins ou non, $\left(n^{\circ} 7\right)$,

— un conseiller directement $\left(n^{\circ} 4 \& 5\right)$.

Cependant, il faut noter que $21 \%$ des configurations de conditions d'apprentissage se déroulent en autonomie complète (configurations $6,9,10$ ) ou quasi-complète (8). Est-ce une réalité ? Deux biais ont pu être introduits :

1. l'utilisation de récits rétrospectifs,

2. le choix d'une population d'agriculteurs minoritaires, qui d'une part sont déjà autonomes dans leurs formes d'expérience, et qui mettent en place des changements « rares et innovants » qui font peu l'objet de références du conseil agronomique actuel.

Contrairement à toutes les autres configurations-types, les configurations 7 «Le réseau de dialogue et la qualité de l'activité », et 8 «Essai-erreur pour améliorer l'état du milieu compatible avec l'organisation du travail» ont des taux d'adoption des changements inférieurs à $50 \%$ (de plus cette modalité «changements non adoptés » apparaît significativement dans l'analyse statistique). Il semble que ces configurations-types soient corrélées à des changements de pratiques plus innovants que les autres (par exemple, le désherbage mécanique, l'introduction de nouvelles cultures), sortant des cadres classiques du conseil agricole, avec moins de références disponibles, et qui sont perçus comme sujets à plus de risques d'échec. Néanmoins, dans les récits des agriculteurs, ces «changements testés non adoptés » font partie de leur trajectoire de changement.

Nous avons procédé à une analyse de la répartition des configurations-types d'apprentissage selon des périodes marquées par des évolutions marquantes du contexte pour les agriculteurs, car énoncées, au moins par certains, comme des éléments qui ont été à l'origine d'un processus d'enquête (Tableau 4 , en annexe).

Quatre grandes périodes ont été différenciées, de la Réforme de la Politique Agricole Commune de l'Union Européenne (1985-1995) à une période d'anticipation de la réglementation à venir (Plan Ecophyto, directive cadre sur l'eau) de 2006 à 2010. L'évolution de ce contexte est marquée par une pression de plus en plus forte des injonctions environnementales. Le tableau 4 met en évidence qu'il coexiste une diversité de configurations de conditions d'apprentissage pour une même période, mais également permet de noter que cette diversité évolue au fil du temps. Ce tableau montre aussi que les configurations où le GDA est un élément important des phases d'expérimentation et d'évaluation, très présentes lors de la première période (environ $50 \%$ ), disparaissent progressivement au profit de configurations de conditions d'apprentissage où les agriculteurs se reposent plus sur eux-mêmes ou le réseau de pairs (50\% des configurations types d'apprentissage de ces agriculteurs dans les deux dernières périodes).

Le tableau 5 permet de constater que, dans un contexte territorial relativement similaire pour les agriculteurs (du point de vue des caractéristiques pédoclimatiques mais aussi des 
ressources disponibles sur le plan à la fois technique, relationnel, économique), la construction de la situation problématique s'avère différente, puisqu'aux différentes époques ceux qui opèrent des changements peuvent le faire en recourant à des configurations-types différentes.

\begin{tabular}{|c|c|c|c|c|c|}
\hline & $1985-1995$ & $1996-2000$ & 2001-2005 & 2006-2010 & \\
\hline Nom de l'agriculteur & Réforme de la PAC & $\begin{array}{l}\text { Montée en puissance } \\
\text { des mesures agro- } \\
\text { environnementales }\end{array}$ & $\begin{array}{l}\text { Éco- } \\
\text { conditionnalité, } \\
\text { contrats territoriaux } \\
\text { d'exploitation }\end{array}$ & $\begin{array}{l}\text { Anticipation de la } \\
\text { réglementation à } \\
\text { venir (Plan } \\
\text { Ecophyto, directive } \\
\text { cadre sur l'eau) }\end{array}$ & $\frac{\mathrm{Nb} \text { total }}{\text { de CCA }}$ \\
\hline Luc & & $\begin{array}{l}3 \text { (deux fois), } \\
7\end{array}$ & $\begin{array}{l}\text { 7(quatre fois), } 2,4, \\
5,10\end{array}$ & 5 & $\underline{12}$ \\
\hline Patrick & $\begin{array}{l}2 \text { (deux fois), } \\
4\end{array}$ & $\begin{array}{l}4 \text { (deux fois), } \\
3,8\end{array}$ & $\begin{array}{l}4 \text { (deux fois), } \\
2,10\end{array}$ & 9 & $\underline{12}$ \\
\hline Jean-Paul & $\begin{array}{l}2 \text { (cinq fois), } \\
1 \text { (deux fois), } \\
7 \text { (deux fois), } \\
3,4,5,6\end{array}$ & $\begin{array}{l}2 \text { (deux fois), } \\
7 \text { (deux fois), } 5\end{array}$ & $\begin{array}{l}2 \text { (deux fois), } \\
4 \text { (deux fois), } \\
7 \text { (deux fois), } \\
8 \text { (deux fois), } \\
1,5,10\end{array}$ & $3,5,6,7,8$ & $\underline{34}$ \\
\hline Pierre & $\begin{array}{l}2 \text { (trois fois), } 3 \\
\text { (deux fois), } \\
1,4,5\end{array}$ & 1 & 4 & $\begin{array}{l}4 \text { (deux fois), } \\
9\end{array}$ & $\underline{13}$ \\
\hline Fabien & 1,3 & 2 & $\begin{array}{l}4 \text { (deux fois), } \\
7 \text { (deux fois), } \\
8,9\end{array}$ & $4,7,8$ & $\underline{15}$ \\
\hline Jean-Christophe & 7,1 & & $7,9,10$ & $\begin{array}{l}9 \text { (quatre fois), } \\
4 \text { (deux fois), } \\
7,8,10\end{array}$ & $\underline{14}$ \\
\hline Francis & $\begin{array}{l}2 \text { (deux fois), } \\
4 \text { (deux fois), } \\
6 \text { (deux fois) }\end{array}$ & 7,8 & $\begin{array}{l}6 \text { (deux fois), } \\
4\end{array}$ & $4,5,7$ & $\underline{14}$ \\
\hline Bruno & 6 & 6 & & $\begin{array}{l}7 \text { (deux fois), } \\
4\end{array}$ & $\underline{5}$ \\
\hline
\end{tabular}

Tableau 5 : Configurations-types de conditions d'apprentissage (CCA) mobilisées par chaque agriculteur selon quatre grandes périodes différentes. En

bleu, les CCA se déroulant en autonomie complète ou quasi-complète

Table 5: Standard configurations of learning conditions (CCA) mobilized by each farmer in four major periods. In blue, the CCA takes place in complete or almost complete autonomy

\section{4.- Discussion et conclusion}

\section{1.- Limites de la méthodologie}

Bien que nous ayons travaillé à l'objectivation de l'évolution des pratiques des agriculteurs avec leurs relevés de pratiques, la reconstruction a posteriori des modalités d'apprentissage présente évidemment les limites bien connues de ce type d'approche faisant appel à la mémoire (Bourdieu, 1986 ; Demazière, 2007). Si certains détails sont nécessairement omis, si certains échecs ou hésitations restent dans l'ombre, nous pouvons néanmoins avoir accès à des récits permettant de reconstruire les ressources que les agriculteurs se souvenaient avoir mobilisées pour réaliser un changement de pratiques particulier, sous la forme de configurations de conditions d'apprentissage. Il a déjà été mentionné dans des travaux de didactique professionnelle que la reconstruction de récits a posteriori se fait relativement aisément par des agriculteurs. En effet, Mayen (2008) note que «les agriculteurs parviennent assez aisément à énoncer une part de leurs stratégies pour résoudre des problèmes, utiliser les ressources humaines dans leur entourage, dans les structures professionnelles. Ils peuvent dire comment ils « trient « et réorganisent les sources potentielles d'influence. En termes de structure conceptuelle, les caractéristiques des ressources «sociales » pour aider à l'action constituent une part des entités de la structure conceptuelle qu'ils ont identifiée et qui 
composent un pan important de leur propre représentation ».

Une seconde limite de notre travail est notre capacité à construire une trajectoire de l'environnement partiellement objectivée pour mieux saisir comment elle se traduit éventuellement dans les types de configurations identifiées et l'intérêt d'une telle analyse. Si ce qui fait situation potentielle de développement est la rencontre d'une trajectoire de l'environnement et une trajectoire du sujet (Mayen, 2008), comment appréhender cette trajectoire de l'environnement et à quel grain le faire ? Bien que nous ayons cherché à identifier des phases permettant de caractériser des évolutions dans l'environnement politique et économique des exploitations, il est certain qu'il manque d'autres éléments qu'on peut imaginer partagés entre les agriculteurs, comme l'offre disponible en ressources matérielles et cognitives dans la zone d'étude par exemple. D'autre part, il y a très probablement des effets de contexte, liés aux trajectoires des individus et liés au milieu qui influent dans les récits des agriculteurs et qui n'ont été que partiellement explorés dans le travail mené.

\section{2.- Interprétation des résultats}

Dans ce travail, nous avons identifié (Tableau 3) des configurations-types de conditions d'apprentissage, définies comme des façons d'apprendre caractérisées par des modalités particulières à chaque phase du processus d'apprentissage (mise en alerte, expérience, évaluation) et qui peuvent donc être communes à plusieurs agriculteurs. Nos résultats montrent également que chaque agriculteur mobilise, au fil de sa carrière, plusieurs configurations-types: ces configurations-types ne sont donc pas des «styles» d'apprentissage propres à chaque agriculteur contrairement à ce que d'autres travaux tentent d'appréhender (Kilpatrick \& Johns, 2003).

La lecture des résultats sur les configurations-types de conditions d'apprentissage met en avant le fait que l'enquête pour rechercher de nouvelles solutions s'exerce avant tout sur des problèmes détectés dans l'exploitation et que l'agriculteur tente de résoudre par lui-même. Ainsi, dans la mise en place de systèmes économes en intrants, les agriculteurs ont appris «par l'expérience» (Kolb, 1984) autant qu'avec autrui. Nos résultats montrent aussi que la forme d'expérience adoptée est très peu discriminante des configurations types de conditions d'apprentissage. Néanmoins, la modalité «expérimentation en comparaison synchronisée », qui se rapproche le plus de l'expérimentation scientifique, est particulièrement pratiquée dans les configurations-types 1 et 4, c'est-à-dire, lorsqu'il y a eu, à un moment donné du processus, une interaction avec un conseiller (quelle que soit sa structure d'appartenance). Dans ces configurations, le taux d'adoption du changement testé est proche de $100 \%$. Mais quand le conseiller est très présent sur l'ensemble des phases comme dans la configurationtype 5 «Influence forte du conseiller», le déroulement de l'expérience est avant tout «l'observation à l'extérieur» et le taux d'adoption est moyen (50\%). Ainsi, l'expérience par simple observation, sans action dans le cadre d'une expérimentation, alors que le conseiller a orienté le choix de la situation à aller observer (ce qui constitue une démarche classique du conseil), n'est pas suffisamment convaincante pour la mise en place du changement ou ne fournit pas suffisamment de preuves tangibles pour l'adoption du changement. Il n'y a peut-être pas eu alors un travail suffisant dans la façon d'analyser l'analogie entre deux situations : la parcelle de l'agriculteur, et celle qui sert de base à la démonstration du conseiller.

Nous constatons aussi qu'au fil du temps, les configurations-types d'apprentissage mobilisant le GDA deviennent moins présentes (Tableau 4). Ceci peut s'interpréter de différentes façons. Soit le GDA a perdu de son importance, voire de sa crédibilité au cours du temps, ce qui rejoindrait le constat fait souvent que ce type de dispositif de conseil a perdu de son importance sur le terrain (Lémery, 2006). Cependant, il est aussi possible d'y voir un effet d'apprentissage des agriculteurs quant à la façon de mobiliser des ressources 
disponibles: ainsi, la place du conseiller a évolué, d'une position centrale dans la construction des formes d'expérience et de critères d'évaluation à une position de personne ressource pour identifier des pistes de solutions (phase de mise en alerte), les agriculteurs ayant acquis une certaine autonomie dans la façon d'expérimenter et d'évaluer des solutions. Ceci est corroboré par les entretiens que nous avons faits avec les agriculteurs qui sont «passés » par le travail avec le GDA. Une dernière interprétation qui est également corroborée par le discours des agriculteurs de notre échantillon est que les structures classiques de développement, Chambres d'Agriculture ou coopératives agricoles, n'ont pas aujourd'hui particulièrement d'éléments sur certains changements de pratiques que souhaitent mettre en œuvre les agriculteurs (nouvelles espèces, couverts d'interculture...). Ces derniers se trouvent donc d'une certaine façon dans l'obligation de puiser, dans leur propre expérience ou dans celles de leurs pairs, les ressources nécessaires pour apprécier les modalités de mise en œuvre et la pertinence de la solution qu'ils testent.

Il est intéressant de noter que les agriculteurs mobilisent plusieurs façons d'opérer l'enquête au cours de leur carrière professionnelle (Tableau 5) pour faire face à l'indétermination liée à une situation problématique. Cela suggère que les agriculteurs construisent aussi au fil du temps de nouvelles façons de conduire leur enquête et de construire leur jugement. De fait, nous pouvons souligner que les agriculteurs qui mobilisent une diversité de configurations d'apprentissage sont aussi ceux qui ont été le plus loin dans l'exploration et la mise en œuvre de pratiques permettant de réduire les intrants (Chantre, 2011).

Enfin, la place importante des configurations-types qui se déroulent en autonomie $(6,8,9$, 10), évoquée auparavant, révèle deux phénomènes du point de vue des agriculteurs :

— un isolement de certains agriculteurs (Jean-Christophe, Francis) dont les changements de pratiques répondront à une logique d'efficience (améliorer l'usage des intrants dans une logique technico-économique) ou de substitution (remplacement de l'usage de produits chimiques par l'usage de produits d'origine biologique ou par une lutte mécanique),

— de façon contrastée, d'autres agriculteurs opèrent ainsi car ils ont développé une capacité à combiner apprentissages en groupe et apprentissages en autonomie grâce à des compétences méthodologiques acquises par le passé lors d'expérimentations collectives (les autres agriculteurs).

\section{3.- Positionnement des résultats en didactique professionnelle et en ergonomie}

L'analyse que nous avons conduite montre tout d'abord que les agriculteurs de notre échantillon évoquent peu la formation continue comme ressource mobilisée pour engager un changement de pratique au niveau des modes de production qu'ils développent. Ainsi, c'est avant tout au sein d'interactions sociales de proximité que celle-ci soit géographique (agriculteurs voisins ou conseillers locaux de terrain) ou éventuellement identitaire (pairs engagés eux-mêmes dans des changements de pratiques, experts dont les compétences sont reconnues sur des techniques alternatives) que les agriculteurs puisent les ressources nécessaires pour enclencher un changement, voire identifient ce qu'il pourrait apporter dans l'exploitation à la fois sur le plan économique ou sur le plan sans doute plus identitaire (qualité de l'activité). Ainsi, c'est bien par une implication dans des communautés de pratiques (Lave \& Wenger, 1991) mais peut-être aussi épistémiques que les agriculteurs développent leur activité comme l'évoque Compagnone (2014) à propos du changement de pratiques, entre autres, pour limiter l'usage des pesticides, en viticulture.

Cependant, les changements résultent rarement de ces seules interactions sociales: elles s'accompagnent de formes d'expériences parfois structurées au sein même de ces communautés ou groupes. Elles sont aussi souvent fruit d'une démarche personnelle pour faire face à des problèmes identifiés dans l'exploitation, en particulier quand il s'agit de problèmes liés aux états du milieu ou aux résultats obtenus sur la culture. Ainsi, c'est 
souvent seul que l'agriculteur expérimente une technique sans nécessairement avoir toujours les clés pour en apprécier l'intérêt à moyen terme. On pointe ainsi la nécessaire reconstruction d'un rapport de continuité à l'expérience déjà évoquée par Mayen et Olry (2012) au sujet de la formation en alternance. Fondamentalement, les formes de l'expérience révèlent deux processus qui sont en jeu dans l'exploration de nouvelles pratiques. D'une part, dans la mesure où ces pratiques impliquent de nouveaux artefacts, elles sont la manifestation des processus d'instrumentation et d'instrumentalisation de ces artefacts (Béguin \& Rabardel, 2000 ; Coquil, 2014 ; Rabardel, 1995). D'autre part, elles se révèlent aussi être un processus d'élaboration de nouvelles règles de construction du jugement en créant des conditions de «suspension du jugement». Une réflexion serait certainement nécessaire pour mieux comprendre ce qui peut aider ce processus afin de reconstruire des règles pour juger de la façon dont une technique expérimentée "marche ». Il s'agit ici d'aider à produire une interprétation qui puise à la fois dans l'expérience de l'agriculteur et dans la situation pour dire «que cela marche ». C'est en partie ce que suggère la didactique professionnelle quand elle invite à mobiliser le récit et la réflexivité (Pastré, 2005). Cette piste nécessiterait toutefois que les conseillers agricoles ou les pairs qui travaillent ensemble développent des formes de mises en récit qui aident à reconstruire ce couplage entre l'expérience et la situation.

Notre analyse, en montrant l'existence d'un nombre restreint mais néanmoins divers de configurations d'apprentissage qui mêlent différemment interaction avec autrui, déroulement de l'expérience, et analyse réflexive, contribue donc à éclairer la façon dont les agriculteurs «utilisent» le potentiel de développement des situations de travail (Mayen, 1999) pour explorer et stabiliser des changements dans leurs pratiques agricoles pour agir dans et sur leur environnement. Les configurations identifiées ne caractérisent pas un style d'apprentissage d'un agriculteur. Potentiellement, elles peuvent en effet toutes être mobilisées au cours de la carrière professionnelle de certains agriculteurs. Certaines coexistent aussi à une même époque, ce qui indique que les agriculteurs mobilisent différemment les ressources présentes dans un même contexte technique et social local. Arriver à mieux saisir cette diversité peut être particulièrement utile pour les conseillers agricoles qui souhaitent accompagner les agriculteurs et leur permettre ainsi d'ajuster leur façon de travailler pour s'inscrire à bon escient dans les processus d'enquête des agriculteurs et dans ce qui leur permet de construire la continuité de leur expérience. Si la didactique professionnelle met surtout en avant les situations et le processus de didactisation pour la formation, nous mettons en lumière la diversité des façons dont ces situations sont constituées en ressources et contribuons à éclairer comment se construisent des zones de proche développement des personnes dans «l'espace de convergence des caractéristiques en évolution d'une situation (de sa trajectoire) et de trajectoires collectives (du métier) et individuelles » (Mayen, 2008).

\section{4.- Quelques recommandations concrètes pour le conseil agricole dans le contexte du projet d'Agroécologie}

Nous avons pu constater par ailleurs que la mobilisation d'une grande diversité de configurations types d'apprentissage a sans doute été nécessaire pour aller vers une réduction radicale et durable de l'usage des intrants (Chantre, 2011). Ce constat invite à proposer aux conseillers dont l'activité est motivée par un changement de pratiques des agriculteurs en faveur de la réduction d'intrants à inciter les agriculteurs ${ }^{10}$ à diversifier leur façon de combiner «recours à autrui » et «recours à l'expérience». D'autres éléments doivent également être pris en compte par ces conseillers agricoles d'après nos résultats. Ainsi, la mise en récit des essais-erreurs dans la trajectoire des agriculteurs peut permettre au

10 Certains conseillers ne sont pas nécessairement aujourd'hui dans cette logique, par exemple dans certaines structures (firmes, négoces, coopératives) qui associent le conseil et la vente d'intrants. 
conseiller d'en tirer parti dans les changements à venir pour travailler par exemple sur les conditions de réalisation de ces essais et sur les critères d'évaluation mobilisés. Nous avons également constaté le faible impact, en termes de changement adopté, des visites faites par les agriculteurs d'expérimentations ou d'expériences réalisées par d'autres. Ce constat devrait inciter les conseillers qui s'appuient sur ces observations à s'interroger sur la façon dont ils accompagnent le processus d'analogie entre la parcelle de démonstration et la parcelle de l'agriculteur et à mieux définir ce qui peut rendre un tel changement pertinent pour l'agriculteur.

L'occurrence simultanée d'interactions fortes avec un conseiller et d'une forme d'expérience se rapprochant de l'expérimentation scientifique est celle qui assure plus certainement l'adoption définitive d'une nouvelle pratique introduite. Pourtant, cette configuration n'est finalement pas la plus fréquente dans le monde du conseil en agriculture aujourd'hui, les formes classiques d'expérimentation dans des stations dédiées et selon des protocoles peu reproductibles par les agriculteurs demeurant privilégiés. Souvent l'agriculteur est livré à luimême pour «se faire la main », les conditions d'évaluation restent peu précisées alors même que la complexité des processus agro-écologiques implique une rigueur dans l'analyse des résultats (effets systémiques à prendre en compte). Le conseiller a donc tout intérêt à promouvoir des modes d'expérimentation à la ferme comme l'expérimentation en comparaison synchronisée, et l'expérimentation collective (qui peuvent d'ailleurs être combinées). Ces dernières semblent efficaces pour aider les agriculteurs de s'engager dans une trajectoire permettant d'aller vers la reconception de leurs systèmes pour réduire l'usage des engrais et des pesticides. L'expérimentation collective multiplie les cas de figure et pose ainsi des questions de généricité des résultats, favorable non seulement à l'apprentissage méthodologique des agriculteurs, mais aussi à un gain de sens critique et d'autonomie pour des apprentissages ultérieurs. Pourtant la mise en avant d'expérimentations collectives ne semble pas à la mode au sein des chambres d'agriculture qui développent de plus en plus une activité de diagnostic individuel. Cette valorisation de l'expérimentation collective et l'ensemble de ces propositions ne pourraient-ils trouver leur place dans le travail d'accompagnement réalisé au sein du réseau de fermes mis dans le cadre du plan Ecophyto qui sera révisé en 2014-2015 pour la période 2016-2021?

\section{REMERCIEMENTS}

Nous tenons à remercier Liz Libbrecht pour la relecture de l'anglais.

\section{BIBLIOGRAPHIE}

Béguin, P. (2004). Mondes, monde commun et versions des mondes. Bulletin de Psychologie, 57 1(469), 45-48.

Béguin, P., Dedieu, B., \& Sabourin, É. (2011). Introduction. In Le travail en agriculture : son organisation et ses valeurs face à l'innovation (pp. 11-17). Paris: Éditions L'Harmattan.

Béguin, P., \& Rabardel, P. (2000). Designing for instrument mediated activity. Scandinavian Journal of Information Systems, 12, 173-190.

Bentley, J. W. (2006). Folk experiments. Agriculture And Human Values, 23(4), 451-462.

Berkes, F., \& Turner, N. J. (2006). Knowledge, learning and the evolution of conservation practice for social-ecological system resilience. Human Ecology, 34(4), 479-494.

Bourdieu, P. (1986). L'Illusion biographique. Actes de La Recherche En Sciences Sociales, 62/63, 6272.

Brousseau, G. (1998). Théorie des situations didactiques. Grenoble: La pensée sauvage.

Bruner, J. (1983). Savoir-faire et savoir dire. Paris: Presses Universitaires de France.

Bruner, J. (2000). Culture et mode de pensée. L'esprit humain dans ses auvres. Paris: RETZ.

Cerf, M., Gibbon, D., Hubert, B., Ison, R. L., \& Jiggins, J. (2000). Cow up a tree: knowing and learning for change in agriculture: case studies from industrialised countries. Paris: Quae. 
Chantre, E. (2011). Apprentissages des agriculteurs vers la réduction d'intrants en grandes cultures: Cas de la Champagne Berrichonne de l'Indre dans les années 1985-2010. Thesis (PhD), AgroParisTech, Paris Institute for Life, Food and Environmental Sciences.

Chantre, E., Cerf, M., \& Le Bail, M. (2014). Transitional pathways towards input reduction in French field crop farms. International Journal of Agricultural Sustainability. doi:10.1080/14735903.2014.945316

Chantre, E., Le Bail, M., \& Cerf, M. (2013). Comment évolue l'expérience des agriculteurs engagés dans l'écologisation de leurs pratiques? Education Permanente, Travail et développement professionnel. Construire l'expérience, 2(197-4), 71-82.

Chevassus-au-Louis, B., \& Griffon, M. (2008). La nouvelle modernité: une agriculture productive à haute valeur écologique. Déméter : Économie et Stratégies Agricoles, 14, 7-48.

Compagnone, C. (2014). Les viticulteurs bourguignons et le respect de l'environnement. Revue française de sociologie, 55(2), 319-358.

Coquil, X. (2014). Transition des systèmes de polyculture élevage laitiers vers l'autonomie, Une approche par le développement des mondes professionnels. AgroParisTech, Paris Institute for Life, Food and Environmental Sciences.

Darnhofer, I., Bellon, S., Dedieu, B., \& Milestad, R. (2010). Adaptiveness to enhance the sustainability of farming systems. A review. Agronomy for Sustainable Development, 30, 545-555.

Darré, J.-P. (1985). La parole et la technique: l'univers de pensée des éleveurs du ternois. Paris: L'Harmattan.

Demazière, D. (2007). A qui peut-on se fier? Les sociologues et la parole des interviewés. Langage \& Société, 121-122(3-4), 85-100.

Dewey, J. (1993). Logique. La théorie de l'enquête. Paris: Presses universitaires de France.

Dewey, J. (2004). Comment nous pensons. Paris: Seuil.

Fabre, M., \& Vellas, E. (2006). Situations de formation et problématisation. De Boeck Supérieur.

Garrigou, A., Baldi, I., \& Dubuc, P. (2008). Apports de l'ergotoxicologie à l'évaluation de l'efficacité réelle des EPI devant protéger du risque phytosanitaire : de l'analyse de la contamination au processus collectif d'alerte. Perspectives interdisciplinaires sur le travail et la santé, (10-1). Retrieved from http://pistes.revues.org/2137

Goulet, F. (2008). Des tensions épistémiques et professionnelles en agriculture. Dynamiques autour des techniques sans labour et de leur évaluation environnementale. Revue D'anthropologie Des Connaissances, 2008/2(4), 291-310.

Hocdé, H., \& Triomphe, B. (2006). L'expérimentation en milieu paysan. In CIRAD-GRET (Ed.), Memento de l'agronome (pp. 511-530).

Husson, F., Lê, S., \& Pagès, J. (2009). Analyse de données avec R. Rennes: Presses Universitaires de Rennes.

Jacquet, F., Butault, J.-P., \& Guichard, L. (2011). An economic analysis of the possibility of reducing pesticides in French field crops. Ecological Economics, 70, 1638-1648. doi:10.1016/j.ecolecon.2011.04.003

Jourdan, M. (1997). Développement technique dans l'exploitation agricole et compétences de 1'agriculteur. Performances Humaines et Techniques, 90, 26-31.

Jouzel, J.-N., \& Dedieu, F. (2013). Rendre visible et laisser dans l'ombre. Revue française de science politique, Vol.63(1), 29-49. doi:10.3917/rfsp.631.0029

Kilpatrick, S., \& Johns, S. (2003). How farmers learn: different approaches to change. Journal of Agricultural Education and Extension, 9(4), 151-164.

Kolb, D. A. (1984). Experiential learning: Experience as the Source of Learning and Development. Englewood Cliffs, NJ.: Prentice-Hall, Inc.

Lamine, C. (2011). Transition pathways towards a robust ecologization of agriculture and the need for system redesign. Cases from organic farming and IPM. Journal of Rural Studies, 27(2), 209219. doi:10.1016/j.jrurstud 2011.02 .001

Lamine, C., Meynard, J.-M., Perrot, N., \& Bellon, S. (2009). Analyse des formes de transition vers des agricultures plus écologiques: les cas de l'Agriculture Biologique et de la Protection Intégrée. Innovations Agronomiques, 4, 483-493. 
Lave, J., \& Wenger, E. (1991). Situated learning. Legitimate peripheral participation. Cambridge: Cambridge University Press.

Lémery, B. (2006). Nouvelle agriculture, nouvelle forme d'exercice et nouveaux enjeux du conseil aux agriculteurs. In J. Rémy, H. Brives, \& B. Lémery, Conseiller en agriculture (pp. 235-252). Paris: Éditions Quae.

Mayen, P. (1999). Des situations potentielles de développement. Education Permanente, 139, 87-107.

Mayen, P. (2008). Dix développements sur la didactique professionnelle et le développement. In Y. Lenoir \& P. Pastré (Eds.), Didactique professionnelle et didactiques disciplinaires en débat. Toulouse: Octarès.

Mayen, P., \& Olry, P. (2012). Expérience du travail et développement pour de jeunes adultes en formation professionnelle. Recherche et formation, (70), 91-106.

Nicourt, C., \& Cabaret, P. (2011). Création de normes, innovation sanitaire et éthique des éleveurs ovins bio. In Le travail en agriculture : son organisation et ses valeurs face à l'innovation (pp. 8598). Éditions L'Harmattan.

Pastré, P. (2005). Genèse et identité. In P. Rabardel \& P. Pastré (Eds.), Modèle du sujet pour la conception (pp. 231-259.). Toulouse: Octarès Editions.

Rabardel, P. (1995). Les hommes et les technologies : une approche cognitive des instruments contemporains. Toulouse: Octarès Éditions.

Rhoades, R., \& Bebbington, A. (1991). Farmers as Experimenters. In B. Haverkort, J. V. D. Kamp, \& A. Waters-Bayer (Eds.), Joining Farmer's Experiments. London: Intermediate Technology Publications.

Rogalski, J. (2004). La didactique professionnelle : une alternative aux approches de «cognition située » et «cognitiviste » en psychologie des acquisitions. @ ctivités, 1(2), 103-120.

Sebillotte, M. (1974). Agronomie et agriculture. Essai d'analyse des tâches de l'agronome. Cahier O.R.S.T.O.M. - Sciences Humaines, 24, 3-25.

Vermersch, P. (2007). Bases de l'auto explicitation. Expliciter, 69, 1-31.

\section{RESUME}

«Développer une agriculture plus écologique», injonction prégnante des politiques territoriales actuelles, invite à s'interroger sur les conditions d'apprentissage des agriculteurs en situation de travail. Notre méthodologie, à l'interface entre didactique professionnelle et agronomie, s'appuie sur des entretiens auprès de vingt céréaliers français, qui ont réduit l'usage des pesticides et engrais au cours de leur carrière professionnelle. Pour analyser la configuration de conditions d'apprentissage accompagnant chaque changement de pratiques vers la réduction d'intrants, nous avons conçu un cadre d'analyse distinguant trois phases successives (la mise en alerte, l'expérience, et l'évaluation), en nous inspirant de la théorie de l'enquête de Dewey (1993). Chaque phase du processus d'apprentissage a été caractérisée par un ensemble de variables elles-mêmes déclinées en un ensemble de modalités. Une classification ascendante hiérarchique a permis d'identifier dix types de configurations de conditions d'apprentissage. Chaque agriculteur mobilise, au fil de sa carrière, plusieurs configurationstypes combinant des formes d'expérience et des personnes ressources variées. Si en tendance les agriculteurs ont modifié leurs pratiques dans la proximité avec autrui, certaines configurationstypes se déroulent en autonomie complète ( $20 \%$ des cas) ce qui révèle à la fois un isolement de certains agriculteurs, et une capacité d'autres agriculteurs à combiner apprentissages en groupe et apprentissages en autonomie. L'identification que nous avons faite de configurations de conditions d'apprentissage constitue une approche originale pour traiter des apprentissages en situation de travail et offre des outils pour structurer un travail d'accompagnement des agriculteurs vers des systèmes de culture innovants. 


\section{MOTS-CLES}

conditions d'apprentissage, agriculteurs, changements de pratiques, enquête

Chantre, E., Le Bail, M., \& Cerf, M. (2014). Une diversité de configurations d'apprentissage en situation de travail pour réduire l'usage des engrais et pesticides agricoles. Activités, 11(2), 3-25. http://www .activites.org/v11n2/v11n2.pdf

Article soumis le 5 juin 2013, accepté pour publication le 10 août 2014 


\begin{tabular}{|c|c|c|c|}
\hline $\begin{array}{l}\text { Phase du } \\
\text { processus }\end{array}$ & Variables retenues & Modalités & Signification \\
\hline \multirow[t]{12}{*}{$\begin{array}{l}\text { 1. Phase de } \\
\text { mise en alerte }\end{array}$} & $\begin{array}{l}\text { Contexte politico- } \\
\text { économique externe à } \\
\text { l'exploitation }\end{array}$ & Oui/non & $\begin{array}{l}\text { Oui si le processus s'est fait dans le cadre d'une incitation } \\
\text { politique ou économique externe à l'exploitation, qu'elle soit à } \\
\text { l'échelle territoriale (Fertimieux), nationale ou européenne } \\
\text { (anticipation des réformes de la PAC) }\end{array}$ \\
\hline & \multirow[t]{6}{*}{ Préoccupation interne } & Résultat de la culture & $\begin{array}{l}\text { rendement généralement et plus exceptionnellement la qualité, } \\
\text { comme le taux de protéines. }\end{array}$ \\
\hline & & Etat du milieu & $\begin{array}{l}\text { Présence d'adventices, maladies, réduction des intrants (sous } \\
\text { réserve de considérer que la réduction d'intrants est une } \\
\text { réduction de leur impact sur le milieu), autres...) mais aussi } \\
\text { sur des états du milieu non visés au départ (biodiversité ou } \\
\text { apparition de nuisibles, odeurs) ou des états du milieu } \\
\text { favorables/défavorables au système de culture. }\end{array}$ \\
\hline & & Economie & $\begin{array}{l}\text { Réduction de charges, amélioration ou le maintien du } \\
\text { revenu... }\end{array}$ \\
\hline & & Qualité de l'activité & $\begin{array}{l}\text { Organisation du travail, mais aussi préoccupation pour la } \\
\text { santé, préoccupation environnementale, et enfin plaisir dans le } \\
\text { travail. }\end{array}$ \\
\hline & & $\begin{array}{c}\begin{array}{c}\text { Economie et qualité de } \\
\text { l'activité }\end{array} \\
\end{array}$ & \\
\hline & & Economie et état du milieu & \\
\hline & \multirow[t]{5}{*}{ Interaction avec autrui } & Non & Pas d'interaction avec autrui \\
\hline & & Pair & $\begin{array}{l}\text { Agriculteur, qui peut être un voisin, ami, membre du groupe de } \\
\text { développement, de la CUMA, de la famille... (la proximité } \\
\text { peut être géographique, familiale, amicale, professionnelle). }\end{array}$ \\
\hline & & f & $\begin{array}{lllll}\begin{array}{l}\text { Conseiller (hors } \\
\text { développement) }\end{array} & \text { conseiller/animateur } & \text { de } & \text { groupe } & \text { de }\end{array}$ \\
\hline & & Groupe de développement & $\begin{array}{l}\text { Tout groupe d'agriculteurs se réunissant plusieurs fois par an } \\
\text { (exemple: GDA, CETA, CIVAM). Ce groupe est } \\
\text { généralement animé par un conseiller qui peut aussi avoir un } \\
\text { rôle de formateur. }\end{array}$ \\
\hline & & $\begin{array}{l}\text { Intervention d'un conseiller } \\
\text { extérieur au sein d'un groupe } \\
\text { de développement }\end{array}$ & $\begin{array}{l}\text { la mise en alerte a été réalisée au sein d'un groupe de } \\
\text { développement avec l'intervention d'un conseiller extérieur au } \\
\text { groupe (formateur } \mathrm{PV} \text {, conseiller privé, ingénieur institut } \\
\text { technique, etc.) }\end{array}$ \\
\hline \multirow[t]{5}{*}{$\begin{array}{l}\text { 2. Phase } \\
\text { «d'expérimen } \\
\text { tation }\end{array}$} & \multirow[t]{4}{*}{$\begin{array}{c}\text { Déroulement de } \\
\text { «l'expérimentation » }\end{array}$} & Comparaison synchronisée & $\begin{array}{l}\text { Comparaison de l'ancienne pratique et de la nouvelle sur } \\
\text { l'exploitation en même temps (exemple typique de deux } \\
\text { bandes de culture où l'on compare deux modalités toutes } \\
\text { choses égales par ailleurs). }\end{array}$ \\
\hline & & Directe & $\begin{array}{l}\text { Changement direct, du jour au lendemain, sur toute la surface } \\
\text { concernée (parcelle ou sole) sans comparaison préalable. }\end{array}$ \\
\hline & & Progressive & $\begin{array}{l}\text { Changement progressif, mais sur toute la surface (par exemple, } \\
\text { pour une réduction de dose de pesticide, réduction } \\
\text { incrémentale sur toute la sole de la culture concernée). }\end{array}$ \\
\hline & & $\begin{array}{l}\text { Observation extérieure à } \\
\text { l'exploitation }\end{array}$ & $\begin{array}{l}\text { Observation extérieure à l'exploitation : chez un voisin, dans } \\
\text { une station expérimentale institutionnelle, etc. }\end{array}$ \\
\hline & Interaction avec autrui & Idem phase de mise en alerte & \\
\hline \multirow{13}{*}{$\begin{array}{c}\text { 3. Phase } \\
\text { d'évaluation }\end{array}$} & \multirow[t]{8}{*}{ Critère d'évaluation } & Résultat de la culture & \multirow[t]{4}{*}{ Idem phase de mise en alerte } \\
\hline & & Etat du milieu & \\
\hline & & Economie & \\
\hline & & Economie et état du milieu & \\
\hline & & Organisation du travail & $\begin{array}{l}\text { Nous avons choisi d'extraire l'organisation du travail qui } \\
\text { semble être un critère d'évaluation bien distinct de la qualité } \\
\text { de l'activité dans cette phase du processus d'apprentissage. }\end{array}$ \\
\hline & & $\begin{array}{l}\text { Organisation du travail et } \\
\text { autre }\end{array}$ & $\begin{array}{l}\text { Organisation du travail et autre critère d'évaluation de cette } \\
\text { liste. }\end{array}$ \\
\hline & & Qualité de l'activité et autre & $\begin{array}{l}\text { Qualité de l'activité et/ou autre critère d'évaluation de cette } \\
\text { liste. }\end{array}$ \\
\hline & & $\begin{array}{l}\text { Résultat de la culture et état } \\
\text { du milieu }\end{array}$ & \\
\hline & \multirow{4}{*}{$\begin{array}{l}\text { Référentiel d'évaluation } \\
\text { externe à l'exploitation }\end{array}$} & Non & Pas de référentiel externe à l'exploitation \\
\hline & & Institutionnel & $\begin{array}{l}\text { Références de Chambre d'Agriculture, Coopératives, Institut } \\
\text { technique, (ex) Service régional de la protection de végétaux, } \\
\text { semencier... }\end{array}$ \\
\hline & & Pair & $\begin{array}{l}\text { référentiel issu d'un pair : discussion, observation des parcelles } \\
\text { d'un voisin, etc. }\end{array}$ \\
\hline & & Pair et institutionnel & \\
\hline & Adoption du changement & Oui/non & $\begin{array}{l}\text { Non si le changement a été testé ou observé ailleurs mais n'a } \\
\text { pas été durablement adopté sur l'exploitation. }\end{array}$ \\
\hline
\end{tabular}

Tableau 2 : Liste des variables descriptives et de leurs modalités retenues pour l'analyse statistique

Table 2: List of descriptive variables and their modalities retained for statistical analysis 


\begin{tabular}{|c|c|c|c|c|}
\hline \multirow{2}{*}{$\begin{array}{l}\text { Intitulé } \\
\text { \% dans l'échantillon }\end{array}$} & \multicolumn{3}{|c|}{ Caractéristiques des phases } & \multirow{2}{*}{$\begin{array}{l}\text { Taux } \\
\text { d'adoption des } \\
\text { changements }\end{array}$} \\
\hline & Mise en Alerte & Expérience & Evaluation & \\
\hline $\begin{array}{l}\text { 1. Dans un GDA avec } \\
\text { l'intervention d'un conseiller } \\
\text { extérieur } \\
\qquad 5 \%\end{array}$ & $\begin{array}{l}\text { Inter.autrui : Dans un GDA avec un conseiller } \\
\text { extérieur } \\
\text { Préoccup. interne : économique } \\
\text { (Contexte politico-économique prégnant) }\end{array}$ & $\begin{array}{l}\text { Inter. autrui : Dans un } \\
\text { GDA avec un conseiller } \\
\text { extérieur } \\
\text { (Déroul.: comparaison } \\
\text { synchronisée). }\end{array}$ & Réf. : Pair et institution & $(100 \%)$ \\
\hline $\begin{array}{r}2 . \text { Dans un GDA } \\
16 \%\end{array}$ & $\begin{array}{l}\text { Inter. autrui : Dans un GDA } \\
\text { éventuellement avec un conseiller extérieur au } \\
\text { GDA } \\
\text { Contexte politico-économique prégnant } \\
\text { Préoccup. interne économique }\end{array}$ & $\begin{array}{l}\text { Inter. autrui : Dans un } \\
\text { GDA }\end{array}$ & $\begin{array}{l}\text { Réf. : Pair et institution } \\
\text { Crit.: économique }\end{array}$ & $100 \%$ \\
\hline $\begin{array}{l}\text { 3. Critères économiques } \\
7 \%\end{array}$ & (Préoccup. interne économique) & $\begin{array}{l}\text { (Pas d'inter. autrui) } \\
\text { (Déroul.: direct) }\end{array}$ & $\begin{array}{l}\text { Crit.: état du milieu et } \\
\text { économie }\end{array}$ & $(100 \%)$ \\
\hline $\begin{array}{l}\text { 4. Test autonome d'une idée } \\
\text { provenant d'un conseiller } \\
19 \%\end{array}$ & $\begin{array}{l}\text { Inter. autrui : conseiller } \\
\text { (Préoccup. interne économique ou qualité de } \\
\text { l'activité) }\end{array}$ & $\begin{array}{l}\text { Pas d'inter. autrui } \\
\text { (Déroul. : comparaison } \\
\text { synchronisée) }\end{array}$ & $\begin{array}{l}\text { Réf. soit interne, soit } \\
\text { institutionnel. }\end{array}$ & $96 \%$ \\
\hline $\begin{array}{l}\text { 5. Influence forte d'un } \\
\text { conseiller } \\
\qquad 7 \%\end{array}$ & Inter. autrui : conseiller & $\begin{array}{l}\text { Inter. autrui : conseiller } \\
\text { Déroul.: observation à } \\
\text { l'extérieur. }\end{array}$ & Réf. : institutionnel & $(50 \%)$ \\
\hline $\begin{array}{l}\text { 6. Améliorer les résultats de } \\
\text { la culture de façon autonome } \\
\qquad \begin{array}{l}7 \%\end{array}\end{array}$ & $\begin{array}{l}\text { Préoccup. interne : résultats de la culture } \\
\text { Indépendant du contexte politico-économique } \\
\text { Inter. autrui : non. }\end{array}$ & (Pas d'inter. autrui) & $\begin{array}{l}\text { Crit.: résultats de la } \\
\text { culture }\end{array}$ & $(78 \%)$ \\
\hline $\begin{array}{c}\text { 7. Réseau de dialogue } \\
18 \%\end{array}$ & $\begin{array}{l}\text { Inter. autrui : pair } \\
\text { Préoccup. interne : qualité de l'activité } \\
\text { dominante }\end{array}$ & Inter. autrui : pair & $\begin{array}{l}\text { Réf. : pair } \\
\text { Crit.: : qualité de } \\
\text { l'activité dominant. }\end{array}$ & $42 \%$ \\
\hline $\begin{array}{l}\text { 8. Essai-erreur pour } \\
\text { améliorer l'état du milieu } \\
\text { compatible avec } \\
\text { l'organisation du travail } \\
\qquad \begin{array}{l}7 \% \\
\end{array}\end{array}$ & $\begin{array}{l}\text { Préoccup. interne : état du milieu et } \\
\text { économie } \\
\text { Indépendant du contexte politico-économique } \\
\text { (Inter. autrui : pair ou conseiller) }\end{array}$ & (Pas d'inter. autrui) & $\begin{array}{l}\text { Crit. : organisation du } \\
\text { travail } \\
\text { (Réf. : pair) }\end{array}$ & $12 \%$ \\
\hline $\begin{array}{l}\text { 9. Essai-erreur autonome } \\
\text { pour améliorer l'état du } \\
\text { milieu } \\
\quad 7 \%\end{array}$ & $\begin{array}{l}\text { Préoccup. interne : état du milieu } \\
\text { Indépendant du contexte politico-économique } \\
\text { (Inter. autrui : pair) }\end{array}$ & $\begin{array}{l}\text { Pas d'inter. autrui } \\
\text { Déroul. : mise en place du } \\
\text { changement directe }\end{array}$ & $\begin{array}{l}\text { Réf. : interne } \\
\text { uniquement } \\
\text { Crit. : état du milieu }\end{array}$ & $50 \%$ \\
\hline $\begin{array}{l}\text { 10. Bricolage autonome pour } \\
\text { le rendement } \\
\qquad 7 \%\end{array}$ & $\begin{array}{l}\text { Indépendant du contexte politico-économique } \\
\text { (Préoccup. interne : qualité de l'activité avec } \\
\text { un autre critère) }\end{array}$ & Pas d'inter. autrui. & $\begin{array}{l}\text { Crit. : résultat de la } \\
\text { culture et état du } \\
\text { milieu }\end{array}$ & $(78 \%)$ \\
\hline
\end{tabular}

Tableau 3 : Les dix configurations types de conditions d'apprentissage

Les configurations sont décrites suivant les modalités statistiquement significatives des différentes variables de chaque phase du processus d'enquête.

En gras : Modalité très significative (dont la p.value $<1.10^{-4}$ ) ;

Police normale : Modalité significative $\left(1.10-4<\right.$ p.value $\left.<1.10^{-1}\right)$;

Entre parenthèses : Modalité peu significative (dont la p.value $>1.10^{-1}$, et qui est présente à plus de $50 \%$ dans la variable pour cette configuration-type d'apprentissage). Les variables non mentionnées sont trop peu significatives.

Inter. Autrui : interaction avec autrui ; Préoccup interne : préoccupation interne ; Réf.: référentiel ; Crit. : critère ; Déroul. : déroulement.

\section{Table 3: The ten standard configurations of learning conditions}

The configurations are described according to the statistically significant modalities of the different variables in each phase of the inquiry process.

Bold: Very significant modality (p.value $\left.<1.10^{-4}\right)$;

Normal font: Significant modality $\left(1.10-4<\right.$ p.value $\left.<1.10^{-1}\right)$;

Between brackets: Low significant modality (p.value $>1.10^{-1}$, which is present at more than $50 \%$ in the variable for this type of configuration). Variables not listed are too insignificant.

Inter. Autrui: interaction with others; Préoccup interne: internal preoccupation; Réf:: repository;

Crit.: criterion; Déroul.: course of the experiment 


\begin{tabular}{|c|c|c|c|c|c|}
\hline & 1985-1995 & $1996-2000$ & 2001-2005 & 2006-2010 & \\
\hline & $\begin{array}{l}\text { Réforme de la } \\
\text { Politique Agricole } \\
\text { Commune de } \\
\text { l'Union Européenne } \\
\text { (U.E.) }\end{array}$ & $\begin{array}{l}\text { Montée en puissance } \\
\text { des mesures agro- } \\
\text { environnementales } \\
\text { (mesures européennes } \\
\text { accompagnant la } \\
\text { réforme) }\end{array}$ & $\begin{array}{l}\text { Éco- } \\
\text { conditionnalité des } \\
\text { aides à l'hectare au } \\
\text { niveau européen, } \\
\text { contrats } \\
\text { territoriaux } \\
\text { d'exploitation } \\
\text { (contrats liés à une } \\
\text { politique de l'État) }\end{array}$ & $\begin{array}{l}\text { Anticipation de la } \\
\text { réglementation à } \\
\text { venir (Plan } \\
\text { Ecophyto, directive } \\
\text { cadre sur l'eau de } \\
\text { l'U.E.) }\end{array}$ & Total \\
\hline $\begin{array}{l}\text { 1. Dans un GDA avec } \\
\text { l'intervention d'un conseiller } \\
\text { extérieur }\end{array}$ & 4 & 1 & 1 & & 6 \\
\hline 2. Dans un GDA & 12 & 3 & 4 & & 19 \\
\hline 3. Critères économiques & 4 & 3 & & 1 & 8 \\
\hline $\begin{array}{l}\text { 4. Test autonome d'une idée } \\
\text { provenant d'un conseiller }\end{array}$ & 5 & 2 & 9 & 7 & \\
\hline 5. Influence forte d'un conseiller & 2 & 1 & 2 & 3 & 8 \\
\hline $\begin{array}{l}\text { 6. Améliorer les résultats de la } \\
\text { culture de façon autonome }\end{array}$ & 4 & 1 & 2 & 1 & \\
\hline 7. Réseau de dialogue & 3 & 4 & 9 & 6 & 22 \\
\hline $\begin{array}{l}\text { 8. Essai-erreur pour améliorer } \\
\text { l'état du milieu compatible avec } \\
\text { l'organisation du travail }\end{array}$ & & 2 & 3 & 3 & \\
\hline $\begin{array}{l}\text { 9. Essai-erreur autonome pour } \\
\text { améliorer l'état du milieu }\end{array}$ & & & 2 & 6 & 8 \\
\hline $\begin{array}{l}\text { 10. Bricolage autonome et } \\
\text { rendement }\end{array}$ & 1 & & 7 & 1 & 9 \\
\hline Total & 35 & 17 & 39 & 28 & 119 \\
\hline
\end{tabular}

Tableau 4 : Occurrence des différentes configurations-types d'apprentissage selon quatre grandes périodes (en grisé, nous avons souligné les configurations les plus représentées à une période donnée)

Table 4: Occurrence of the different standard configurations of learning conditions in four major periods (the configurations most frequently represented during a given period are shaded) 